Research Article

\title{
Combined Diagnosis of PD Based on the Multidimensional Parameters
}

\author{
Mohammad Heidari \\ Department of Mechanical Engineering, Abadan Branch, Islamic Azad University, Abadan, Iran \\ Correspondence should be addressed to Mohammad Heidari; moh104337@yahoo.com
}

Received 5 August 2016; Accepted 15 September 2016

Academic Editor: Aiguo Song

Copyright (C) 2016 Mohammad Heidari. This is an open access article distributed under the Creative Commons Attribution License, which permits unrestricted use, distribution, and reproduction in any medium, provided the original work is properly cited.

This paper presents a comprehensive multiparameter diagnosis method based on multiple partial discharge (PD) signals which include high-frequency current (HFC), ultrasound, and ultrahigh frequency (UHF). The HFC, ultrasound, and UHF PD are calculated under different types of faults. Therefor the characteristic values, as nine basic characteristic parameters, eight phase characteristic parameters, and the like are calculated. Diagnose signals are found with the method based on information fusion and semisupervised learning for HFC PD, adaptive mutation parameters of particle entropy for ultrasonic signals, and IIA-ART2A neural network for UHF signals. In addition, integrate the diagnostic results, which are the probability of fault of various defects and matrix, of different PD diagnosis signals, and analysis with Sugeno fuzzy integral to get the final diagnosis.

\section{Introduction}

Only part of the power equipment insulation generates a discharge. The effect that a fixed discharge channel is not formed in the discharge area is called PD. The PD can reflect the presence of insulation faults in the power equipment. The development of PD will speed up the insulation deterioration of the internal power equipment, leading to internal electrical equipment insulation fault occurrence, resulting in equipment fault and reducing the reliability of power supply. So it is vital to monitor the existence of PD in electrical equipment, as well as the type of discharge and early detection of equipment insulation faults, to deal with it in time, and to avoid the failure of electrical equipment and reduce the serious economic losses caused by power equipment insulation fault.

Kim et al. [1] examined an intelligent spacer built into the internal type UHF PD sensors. Three-dimensional electromagnetic simulations were performed to analyze electricfield distribution of the single-phase GIS and three-phase GIS. After considering the spacer's specifications, sensor structures were designed and analyzed using the 3D EM simulator.

Kaneko et al. [2] used a method for diagnosing the insulation of a gas-insulated switch gear which is to detect PDs using signals in the UHF band.
Schwarz et al. [3] presented that the aim was the detection of beginning destruction in the electrical insulation as a result of electrical stress. A multiplicity of different PD sources and their appearances show different physical and electrical characteristics [4].

Sahoo et al. [5] presented PD detection, measurement, and classification which constitute an important tool for quality assessment of insulation systems utilized in HV power apparatus and cables. The patterns obtained with PD detectors contain characteristic features of the source/class of the respective PD process involved. Gao et al. [6] used the UHF methods for detecting PD owing to its sensitivity and antiinterference capability.

They modeled five types of typical PD sources to imitate the defects in GIS online.

Wu et al. [7] presented a method based on statistical parameters to optimize the algorithm of multi-PD. They used this method for estimation of residual breakdown voltage of generator bars and the optimized multiparameter diagnosis algorithm. Wu et al. [8] used a method to quantitatively determine the reliability of multiparameter diagnosis and to optimize the algorithm of multiparameter diagnosis. Gulski and Kreuger [9] used a computer-aided discharge analyzer, a combination of statistical and discharge parameters to discriminate between different discharge sources. 
Saha [10] presented cellulosic paper and oil insulation in a transformer degrade at higher operating temperature.

In this paper, the available PD charging detection methods include ultrasonic method, UHF method, and HFC method. The combined diagnosis of PD based on the multidimensional characteristic parameters is brought by Sugeno fuzzy integral to process the collected signals. A large amount of experimental data shows that the method for PD signal has well diagnostic accuracy and is able to judge the internal insulation of electrical equipment.

\section{PD Extraction}

2.1. Basic Characteristic Parameters. The basic parameters of $\mathrm{PD}$ are as follows.

(A) Maximum PD Quantity max Q. The maximum discharge quantity within one sample data is

$$
\max Q=\max \left\{Q_{i}\right\} .
$$

(B) Average PD Quantity avg $Q$. The total discharge quantity within cycle/discharging times is

$$
\operatorname{avg} Q=\operatorname{mean}\left\{Q_{i}\right\} .
$$

(C) Average Discharge Current I. The discharge quantity per unit time (one cycle) is

$$
I=\frac{1}{T} \sum_{i} Q_{i}
$$

(D) Mean Square Rate. The mean square value of PD pulse discharge quantity per unit time (one cycle) is

$$
D=\frac{1}{T} \sum_{i} Q_{i}^{2}
$$

(E) NQN. This parameter reflects the number of PD pulses and the discharge intensity.

$$
\mathrm{NQN}=\frac{F S}{G \cdot N}\left[\frac{\log _{10} P_{1}}{2}+\sum_{i=2}^{N-1} \log _{10} P_{i}+\frac{\log _{10} P_{N}}{2}\right] .
$$

In (5), $P_{i}, N$, and $G$ represent the number of pulses per second in the quantization window $I$, the number of amplitude windows, and the gain of PD detector, respectively.

$F S$ is the maximum number of quantization window in the unit $\mathrm{mV}$ gain.

(F) $\max Q 95$. It is the maximum of the discharge quantity with $5 \%$ of the maximum PD quantity of discharge pulse removed.

(G) avg Q95. It is the average of the discharge quantity with $5 \%$ of the maximum PD quantity of discharge pulse removed.
(H) $\max 10, \max 20, \max 50, \max 100, \max X X$. These are the minimum amplitude of $\mathrm{xx}$ maximum $\mathrm{PD}$ within each cycle (in $\mathrm{mV}$ ).

(K) avg max 10, avg max 20, avg max 50, avg max 100, avg max $X X$. These are the average amplitude of $x x$ maximum $\mathrm{PD}$ within each cycle (in $\mathrm{mV}$ ).

The parameters of rank (A) to (E) are values obtained which were assumed in the absence of interference. Actual testing will inevitably introduce severe interference. Therefore, based on a large number of experiments and statistical data, four very valuable parameters of rank $(\mathrm{F})$ to $(\mathrm{K})$ were proposed. A lot of statistics show that, in the field detection with interference, the credibility of the insulation condition reflected by parameters above is greatly improved compared to conventional parameters [11]. Table 1 shows the extraction of partial basic characteristic parameters of PD.

2.2. Phase Characteristic Parameters. When a PD abnormal signal was found, the type of PD needs to be determined. Judging the type of PD is generally based on PD phase distribution spectrums, which mainly include phase distribution of the maximum discharge quantity $H_{q \max }(\Phi)$; phase distribution of the average discharge quantity $H_{q n}(\Phi)$; phase distribution of discharge times $H_{n}(\Phi)$; amplitude distribution of $\mathrm{PD}_{H(q)}$; energy distribution of $\mathrm{PD}_{H(p)}$. There are totally five two-dimensional discharging spectrums and a threedimensional discharging spectrum $H_{n}(\Phi, q)$. Suppose the measured number of fundamental frequency cycle is $m$, and each frequency cycle is divided into $n$ phase windows, with each phase window represented by $\Phi_{i}$; then it is clear that

$$
\begin{aligned}
& H_{q \max }\left(\Phi_{i}\right)=\max \left\{q_{1}\left(\Phi_{i}\right), q_{2}\left(\Phi_{i}\right), \ldots, q_{k}\left(\Phi_{i}\right)\right\}, \\
& H_{\text {qmean }}\left(\Phi_{i}\right)=\frac{\sum_{j=1}^{k} q_{j}\left(\Phi_{i}\right)}{k} .
\end{aligned}
$$

$H_{q n}\left(\Phi_{i}\right)$ is the sum of the discharge numbers in the phase window $\Phi_{i}$, and $H\left(q_{i}\right)$ and $H\left(p_{i}\right)$ are the number of discharge times and energy where the discharge quantities are $q_{i}$ and $p_{i}$, respectively. In (7), $k$ is the discharge number of the phase window $\Phi_{i}$. In this system, $m$ is the maximum number of fundamental frequency cycle intercepted from actual data. Discharge quantity is divided into 25 intervals. According to the above five characteristic spectrums based on phase analysis, PD can be derived from the phase characteristic parameters as shown in Table 2 .

Also

(a) $\mathrm{Sk}^{+}$is defined as below:

$$
\mathrm{Sk}^{+}=\frac{\sum\left(x_{i}^{+}-\mu\right)^{3} \cdot P_{i}}{\sigma^{3}},
$$

in (8), $x, P, \mu$, and $\sigma$ are discrete value, probability of $x$, average value of distribution, and the standard deviation of the distribution, respectively,

(b) the definition of $\mathrm{Sk}^{-}$is in the following:

$$
\mathrm{Sk}^{-}=\frac{\sum\left(x_{i}^{-}-\mu\right)^{3} \cdot P_{i}}{\sigma^{3}},
$$




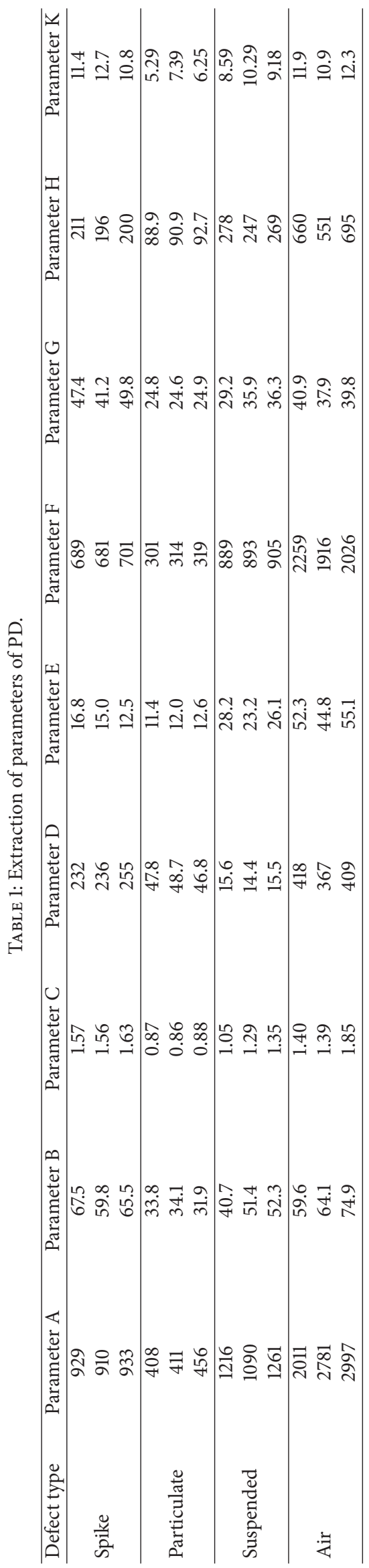


TABLE 2: List of characteristics parameters.

\begin{tabular}{|c|c|c|c|c|c|}
\hline \multirow{2}{*}{ Characteristics } & \multicolumn{5}{|c|}{ PD distribution pattern } \\
\hline & $H_{q \max }(\Phi)$ & $H_{q n}(\Phi)$ & $H_{n}(\Phi)$ & $H(q)$ & $H(p)$ \\
\hline Spectrum skewness $\mathrm{Sk}^{+}$ & $\sqrt{ }$ & $\sqrt{ }$ & $\sqrt{ }$ & $\sqrt{ }$ & $\sqrt{ }$ \\
\hline Spectrum skewness $\mathrm{Sk}^{-}$ & $\sqrt{ }$ & $\sqrt{ }$ & $\sqrt{ }$ & & \\
\hline Spectrum kurtosis $\mathrm{Ku}^{+}$ & $\sqrt{ }$ & $\sqrt{ }$ & $\sqrt{ }$ & $\sqrt{ }$ & $\sqrt{ }$ \\
\hline Spectrum kurtosis $\mathrm{Ku}^{-}$ & $\sqrt{ }$ & $\sqrt{ }$ & $\sqrt{ }$ & & \\
\hline The number of local peaks $\mathrm{Pe}^{+}$ & $\sqrt{ }$ & $\sqrt{ }$ & $\sqrt{ }$ & & \\
\hline The number of local peaks $\mathrm{Pe}^{-}$ & $\sqrt{ }$ & $\sqrt{ }$ & $\sqrt{ }$ & & \\
\hline Discharge asymmetry $Q$ & $\sqrt{ }$ & $\sqrt{ }$ & $\sqrt{ }$ & & \\
\hline Correlation factor $\mathrm{cc}$ & $\sqrt{ }$ & $\sqrt{ }$ & $\sqrt{ }$ & & \\
\hline
\end{tabular}

(c) $\mathrm{Ku}^{+}$is as below:

$$
\mathrm{Ku}^{+}=\frac{\sum\left(x_{i}^{+}-\mu\right)^{4} \cdot P_{i}}{\sigma^{4}}-3,
$$

(d) in the following equation, the definition of $\mathrm{Ku}^{-}$has been presented:

$$
\mathrm{Ku}^{-}=\frac{\sum\left(x_{i}^{-}-\mu\right)^{4} \cdot P_{i}}{\sigma^{4}}-3,
$$

$(e, f)$ the number of local peaks $\mathrm{Pe}^{+}$and the number of local peaks $\mathrm{Pe}^{-}$are the ratio of the pulses' number and the number of phase intervals in positive and negative half cycle of atlas, respectively,

(g) discharge asymmetry $Q$ is the ratio of discharge corresponding parameters' average value in positive half cycle and negative half cycle:

$$
Q=\frac{Q_{S}^{+} / N^{+}}{Q_{S}^{-} / N^{-}}
$$

$Q_{S}^{+}$and $H(\Phi)$ are the sum of corresponding parameter values distributed in the positive half cycle and negative half cycle. $N^{+}$and $N^{-}$are the number of nonzero value distributed in positive half cycle and negative half cycle of the voltage,

(h) correlation factor (cc) is used to assess the difference on shape and distribution between $H^{+}(\Phi)$ and $H^{-}(\Phi)$ [11]:

$$
\mathrm{cc}=\frac{\sum x y-\sum x \sum y / n}{\sqrt{\left[\sum x^{2}-\left(\sum x\right)^{2} / n\right] \cdot\left[\sum y^{2}-\left(\sum y\right)^{2} / n\right]}},
$$

$x$ and $y$ are discharge parameters in positive and negative half-cycle phase window. Also, $n$ is the number of phase window of each half cycle [12]. PD generated by different types of insulation defects have a significant difference in the phase distribution atlas, and the fingerprint database established by the simulation test in laboratory can be taken as a reference in detecting and identifying a defect at the scene. Table 3 shows the PD data phase characteristic parameters.

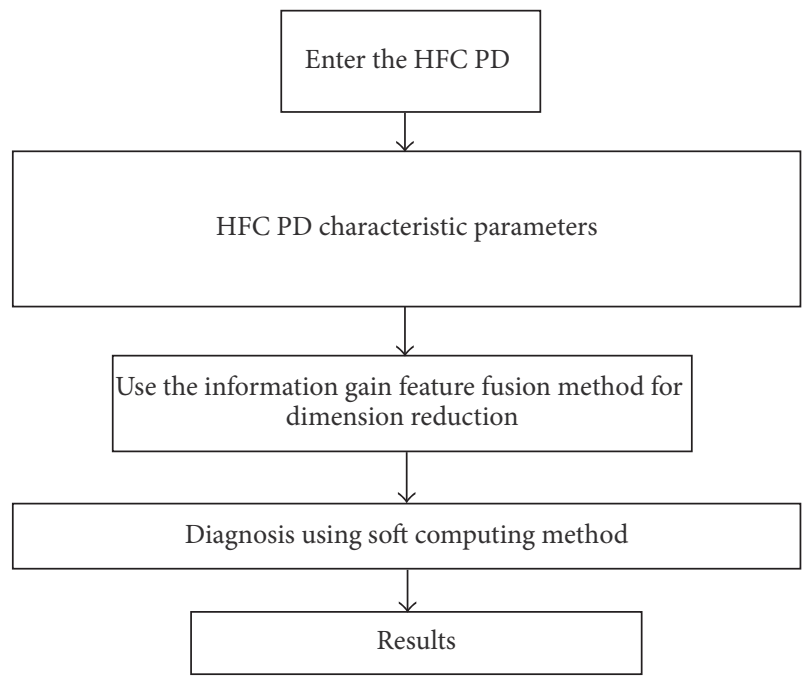

FIgURE 1: The flow chart of diagnosis method.

\section{Single PD Diagnosis Algorithm}

3.1. High-Frequency Current PD Data's Diagnostic Method Based on Information Fusion and Semisupervised Learning. In this paper, for the diagnosis of high-frequency current PD data, a method based on information fusion and semisupervised learning is used. Figure 1 shows the flow chart of this method.

The implementation steps of the algorithm can be described as follows.

Given a data collection $X=\left\{x_{1}, x_{2}, \ldots, x_{l}, x_{l+1}, \ldots, x_{n}\right\}$ and a tag collection $C=\{1,2, \ldots, c\}$, the first $l$ ones $x_{i}(i \leq l)$ are already labeled samples, labeled as $y_{i} \in\{1,2, \ldots, c\}$, and the last $u$ ones $x_{i}(l+1<u \leq n)$ are unlabeled samples. The targets are the labels of predicted unlabeled samples. Define an $n \times c$ matrix $F=\left[F_{1}^{T}, \ldots, F_{n}^{T}\right]^{T} . F_{i}(i \in 1,2, \ldots, n)$ is the energy vector of $x_{i}$ and $x_{i}$ is tagged as $y_{i}=\arg \max _{j<c} F_{i j}$. Define the initial $n \times c$ matrix $Y$. Steps of iterative solver are as follows [13]:

(1) Construct undirected weighted graph $G=(X, E) ; X$ is a collection of samples and $E$ is a set of edges of figure $G$. 


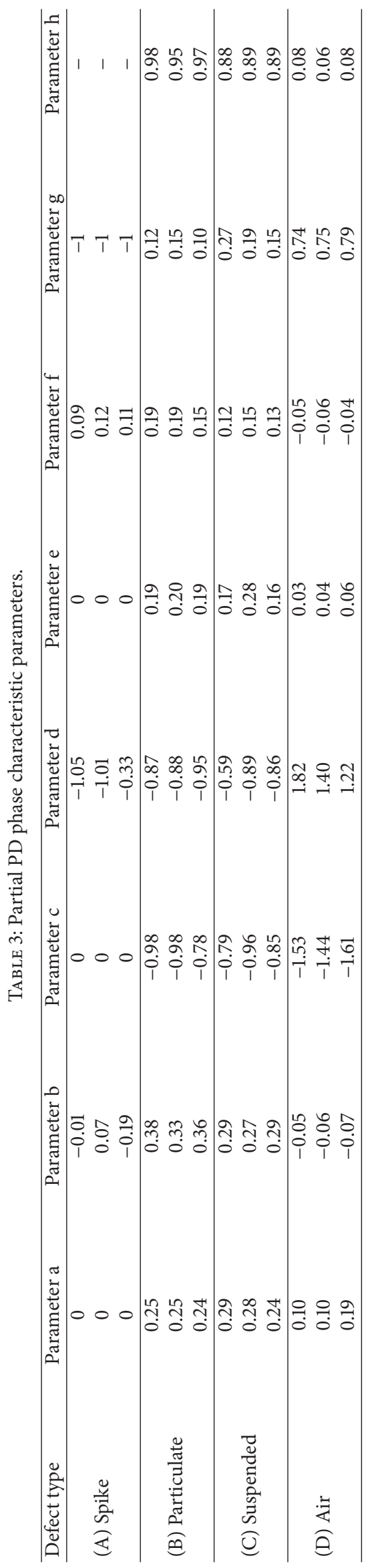


TABLE 4: The recognition result.

\begin{tabular}{lcccc}
\hline Defect type & Spike & Particulate & Suspended & Air \\
\hline Spike & 678 & 234 & 45 & 73 \\
Particulate & 29 & 810 & 33 & 101 \\
Suspended & 31 & 69 & 880 & 37 \\
Air & 60 & 152 & 54 & 730 \\
\hline
\end{tabular}

(2) Construct similar matrix $K$.

(3) Matrix $L$ with $L=D^{-1 / 2} K D^{-1 / 2}$ is calculated and $D$ is

$$
D_{i i}=\sum_{i=1}^{l+u} K_{i j}
$$

(4) The problem is solved by Iterative method.

(5) When $F_{i j}>F_{i k}(k \neq j)$, mark each $x_{i}$ as $y_{i}=j$.

Table 4 shows the four types of discharge model and the recognition result of semisupervised learning. From Table 4, it is clear that the accuracy rate of spike discharge is $79 \%$, particulate discharge is $88 \%$, suspended discharge is $85 \%$, and air discharge is $83 \%$.

3.2. Ultrasonic Signals Diagnostic Method Based on Adaptive Mutation Parameters of Particle Entropy. In this paper, the parameters are optimized with a method based on particle entropy parameter adaptive mutation and then used for PD ultrasound data' diagnosis. Figure 2 shows the flow chart of this method.

Process of parameters' adaptive mutation algorithm based on particle entropy is as follows:

(1) Randomly determine the initial position and velocity of each particle in the data field, and set $E_{0}$ as the stable threshold value of particle entropy.

(2) Set the particles' $p_{\text {best }}$ to the current optimal position, and set $p_{g d}$ as the global optimal position of initial population.

(3) Update the position and velocity of the particles.

(4) If the particle fitness is better than the fitness of $p_{g d}$, update $p_{g d}$ with the current position.

(5) Calculate the collection of particle entropy and determine whether the entropy of each particle is less than the predetermined threshold value $E_{0}$, and perform (6) when it is true, otherwise skip.

(6) Vary $w$ to $w_{0}$, calculate the variation value of $p_{g d}$, and then continue to update the iteration.

(7) Determine whether the convergence criteria of algorithm are met; if true, implement (8); otherwise, continue iterating.

(8) Output $p_{g d}$ and end the algorithm.

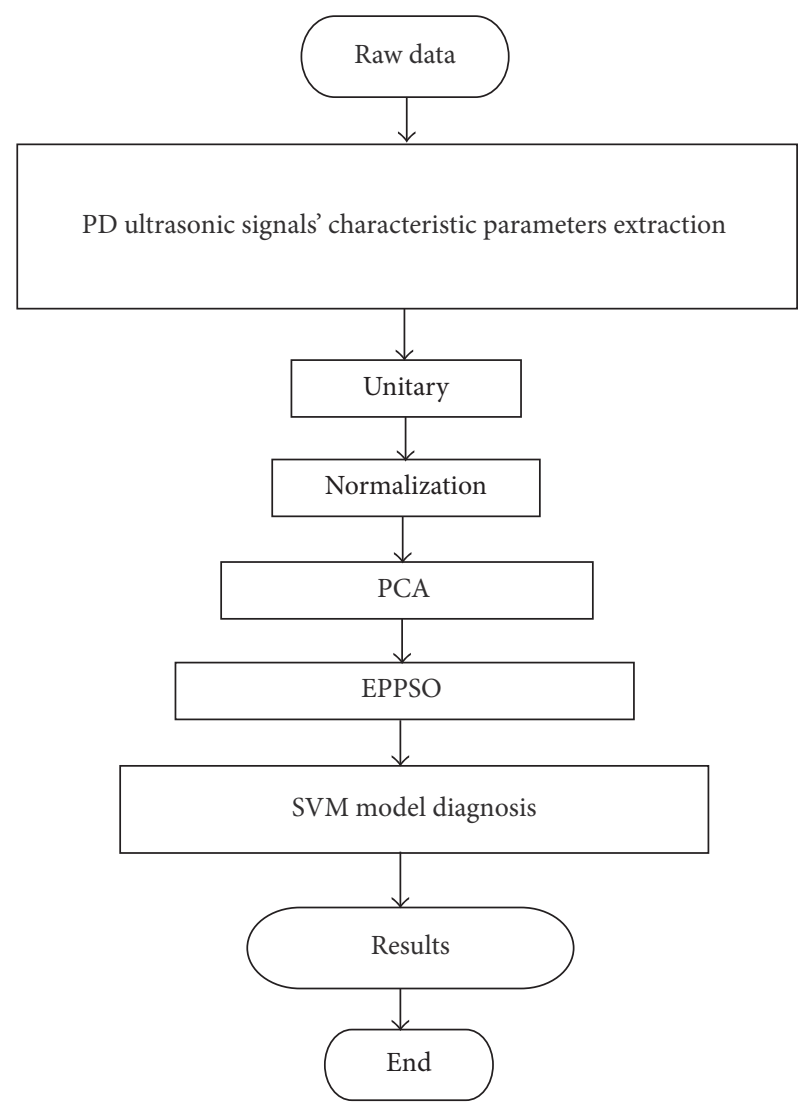

FIGURE 2: Diagnosis with SVM.

3.3. UHF Signal Diagnostic Method Based on IIA-ART2A. Parameters of ART2 neural network (NN) are optimized with a method based on immune algorithm with the improved variation of natural cycle and then used for the UHF PD data diagnostic. Figure 3 shows the flow chart of this method based on NN.

IIA-ART2A NN is unsupervised learning. If we select the correct input vector, only a few samples, then we can get high recognition accuracy $[14,15]$. One thousand new sample data set are selected in each type of defect, characteristic parameters are extracted, and pattern recognition with IIA-ART2A $\mathrm{NN}$ is done [16]. Table 5 shows the recognition results, where the fractal dimension numerical lists only the 4 th node of 4 floor wavelet packet decomposition, and matching nodes 1-4 represent spike, particulate, suspended, and air, respectively. It is clear that the recognition rate of spike discharge is $91 \%$, $\mathrm{PD}$ is $85 \%$, suspended discharge is $84 \%$, and air discharge is $77 \%$.

\section{Multiple Classifier's Fusion Analysis Based on Sugeno Fuzzy Integral}

In this work, a multiple classifier fusion based on Sugeno fuzzy integral is used, to integrate the final diagnosis matrix of different PD diagnosis signals (HFC, ultrasound, and UHF) and calculate the final diagnosis result. Figure 4 shows the flow chart of this strategy. 


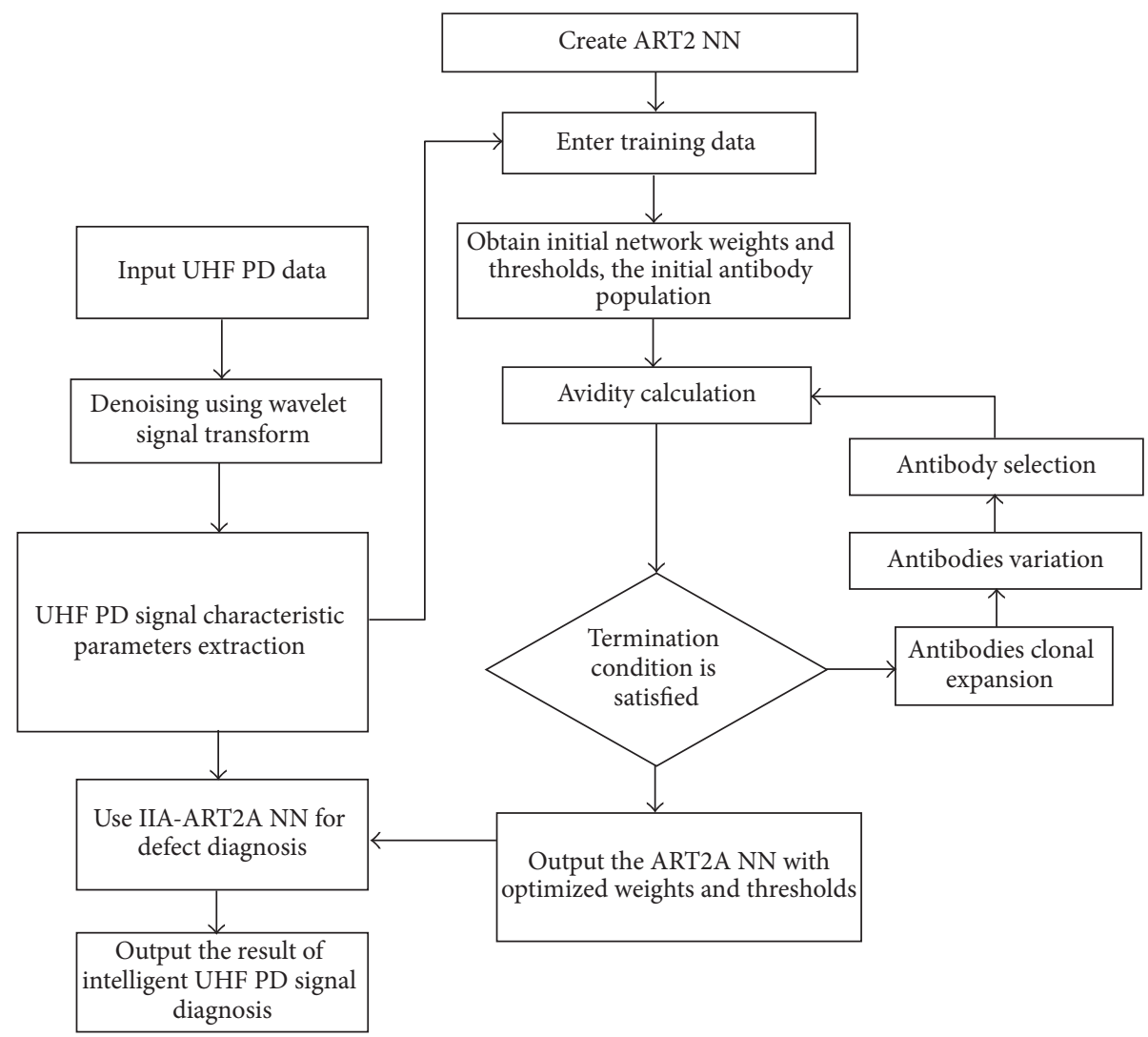

FIGURE 3: The flow chart of UHF signal diagnostic method.

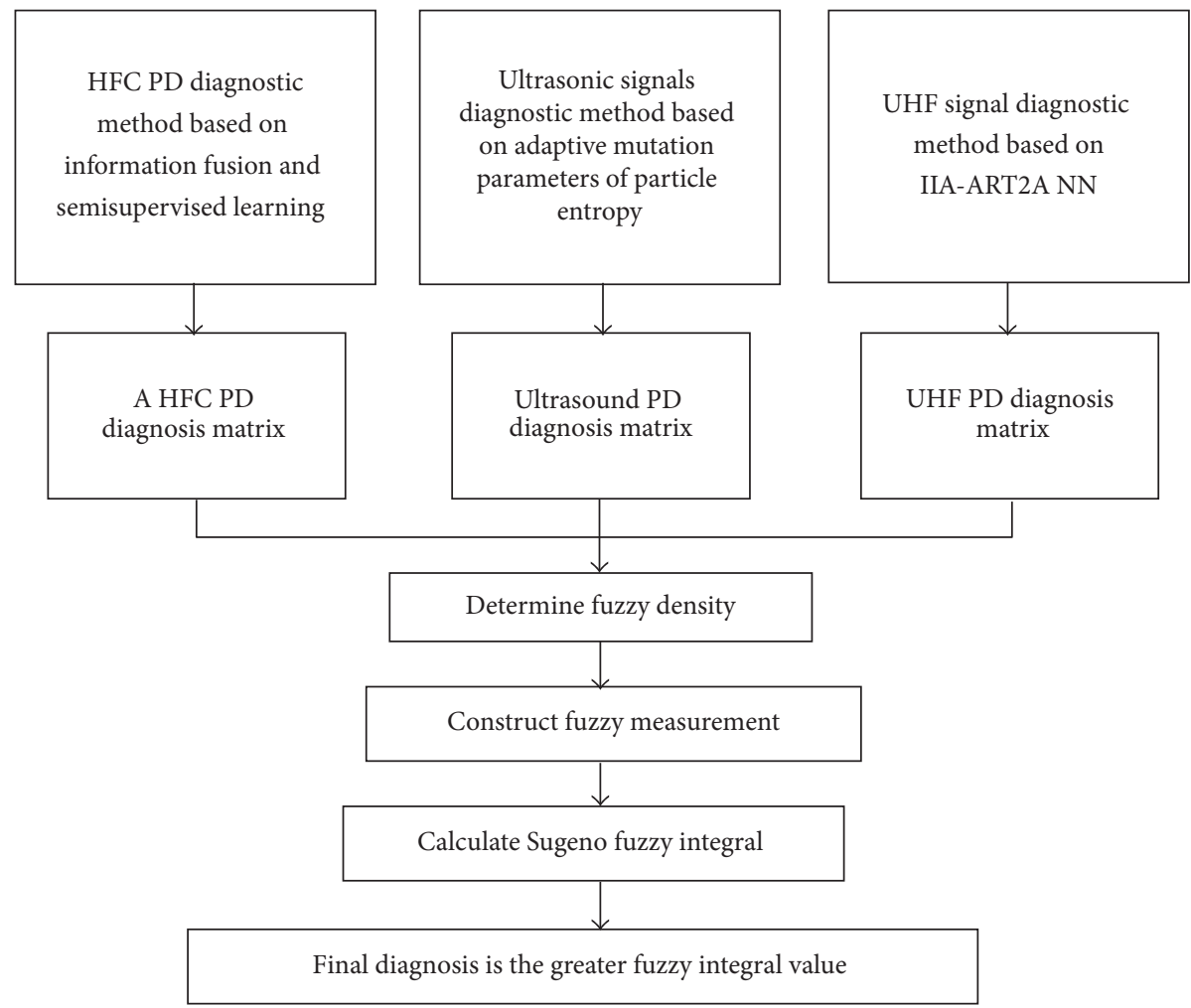

Figure 4: The flow chart of Sugeno fuzzy integral. 
TABLE 5: The recognition result of IIA-ART2A NN.

\begin{tabular}{|c|c|c|c|c|}
\hline Number & Fractal dimension & Damp coefficient & Similarity & Matching node \\
\hline 0 & 0.072 & 0.01 & 0.9918 & 1 \\
\hline 1 & 0.183 & 0.001 & 1 & 2 \\
\hline 2 & 0.183 & 0.001 & 1 & 2 \\
\hline 3 & 0.071 & 0.01 & 0.9972 & 1 \\
\hline 4 & 0.072 & 0.01 & 0.9298 & 1 \\
\hline 5 & 0.098 & 0.02 & 0.9986 & 3 \\
\hline 6 & 0.098 & 0.02 & 0.9996 & 3 \\
\hline 7 & 0.099 & 0.02 & 0.9680 & 3 \\
\hline 8 & 0.081 & 0.01 & 0.9974 & 1 \\
\hline 9 & 0.088 & 0.01 & 0.9972 & 1 \\
\hline 10 & 0.136 & 0.1 & 0.8999 & 4 \\
\hline 11 & 0.122 & 0.1 & 0.9731 & 4 \\
\hline 12 & 0.181 & 0.1 & 0.8997 & 4 \\
\hline 13 & 0.083 & 0.01 & 0.9678 & 1 \\
\hline 14 & 0.092 & 0.01 & 0.9982 & 1 \\
\hline
\end{tabular}

Combining a plurality of different classifiers to obtain high accuracy is an important research topic. There is interaction between the classifiers, rather than being independent. Fuzzy integral is nonlinear integral based on fuzzy measurement, and fuzzy measurement is a nonnegative nonadditive set function, while the nonadditive characteristic of fuzzy measurement can precisely describe interactions between classifiers. Therefore, exploring fusion technology of multiple classifiers based on fuzzy integral is the content of this article. Let $C=\left\{C_{1}, C_{2}, \ldots, C_{n}\right\}$ be a collection of $n$ target categories and $X=\left\{x_{1}, x_{2}, \ldots, x_{m}\right\}$ is a collection of $m$ classifiers. $Z_{k}$ is the $k$ th identified object. After sample $Z_{k}$ is identified by each classifier, we can define a matrix referred to as $\operatorname{PD}\left(Z_{k}\right)$, which is the cross section in decision-making model [12].

$$
\operatorname{PD}\left(Z_{k}\right)=\left[\begin{array}{ccc}
h_{11}^{k} & \cdots & h_{1 n}^{k} \\
\vdots & \ddots & \vdots \\
h_{m 1}^{k} & \cdots & h_{m n}^{k}
\end{array}\right] .
$$

And each row vector $h_{1}=\left(h_{i 1}^{k}, h_{i 2}^{k}, \ldots, h_{i n}^{k}\right), i=1,2, \ldots, m$, is the recognition results of a classifier $x_{i}$ on the sample $Z_{k}$ in various categories, and we call it output vector of classifier $x_{i}$.

Each column vector $h_{j}=\left(h_{1 j}^{k}, h_{2 j}^{k}, \ldots, h_{m j}^{k}\right), j=1,2, \ldots$, $m$, represents recognition results of each classifier on the sample $Z_{k}$ in category $C_{j}$. When the sample is fixed, $h_{j}$ can be thought of as a function $h_{j}: X \rightarrow[0,1]$ (if the output of the classifier is not in the interval $[0,1]$, it can be normalized to meet the conditions), which maps the classifier $x_{i}$ to the corresponding components $h_{i j}$ of fusion vector $C_{j}$. Each intersection $h_{i j}^{k}$ of the output vector $x_{i}$ and the fusion vector $C_{j}$ represents the degree of determination on the fact that the class $x_{i}$ assigned sample $Z_{k}$ to category $C_{j}$, also known as the objective estimate of the determination that $Z_{k}$ belongs to $C_{j}$ of the classifier $x_{i}$. When $h_{i j}^{k}=1$, the classifier $x_{i}$ determines that $Z_{k}$ belongs to the category $C_{j}$; on the contrary, when $h_{i j}^{k}=0, x_{i}$ confirms that $Z_{k}$ does not belongs to category $C_{j}$. Let $g$ be the Sugeno fuzzy measurement of the defined power set $P(X)$ of $X$, fuzzy measurement on a single set of points (the fuzzy density).

$g^{j}=g\left(\left\{x_{i}\right\}\right), i=1,2, \ldots, m$, indicates the credibility of the decisions made by classification $x_{i}$.

$\forall A \in P(X), g(A)$ represents degree of reliability of local decision made by a subset $A$ of $X$. Fuzzy integral fuses the objective evaluation of the determination that $Z_{k}$ belongs to $C_{j}$ (fusion vectors of $C_{j}$ ) and the degree of reliability of classifiers credibility; the integral value is the total objective evaluation of the system that the sample belongs to category $C_{j}$. Thus, the system has integrated value for each category, taking the corresponding category of maximum integrated value as the system's determined category of sample $Z_{k}$.

Therefore, the basic steps of fusion with fuzzy integral can be summarized as follows:

(1) Determine fuzzy density.

(2) Construct fuzzy measurements. Parameters are determined through the fuzzy density, and then fuzzy measurements are also determined.

(3) Calculate fuzzy integral, the integrated extent of determination of the fused object to be identified belonging to each category.

(4) Size comparison of the integral value is carried out, to determine the category of the object to be identified.

In order to facilitate the integration of fuzzy arithmetic, we must first set the output of the probability of failure, provided $w_{i}(i=1,2, \ldots, m)$ the corresponding characteristic parameters' values of sample data and the $m$ th fault; the occurrence probability of $i$ th fault is defined as

$$
\eta_{i}=\frac{w_{i}}{w_{i}+w_{k}}, \quad w_{k}=\underset{k=1,2, \ldots, m}{k \neq i}\left(w_{k}\right) .
$$

And $0<\eta_{i} \leq 1$ and $\eta_{i}$ has a clear sense; it reflects the difference between the binding energy of sample and the $i$ th fault and the binding energy of sample and the fault second to $i$ th. When the two values are equal $\eta_{i}=0.5$, critical state $\eta_{i}>0.5$, it can be classified as $i$ type, and the greater the 
TABLE 6: Accuracy of the classifiers.

\begin{tabular}{|c|c|c|c|c|c|}
\hline Defect model & Testing samples & Training samples & $\mathrm{C} 1$ & $\mathrm{C} 2$ & $\mathrm{C} 3$ \\
\hline (A) Spike & 1000 & 500 & 0.79 & 0.85 & 0.96 \\
\hline (B) Particulate & 1000 & 500 & 0.83 & 0.79 & 0.88 \\
\hline (C) Suspended & 1000 & 500 & 0.89 & 0.78 & 0.84 \\
\hline (D) Air & 1000 & 500 & 0.85 & 0.89 & 0.79 \\
\hline
\end{tabular}

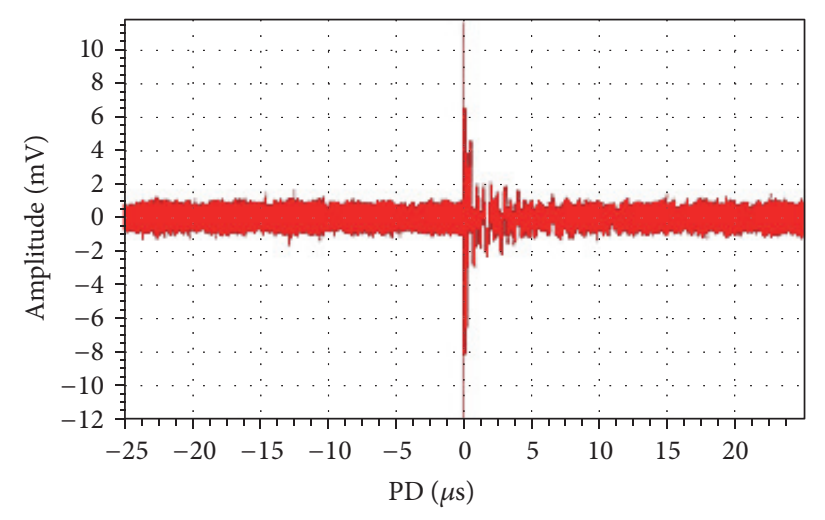

(a)

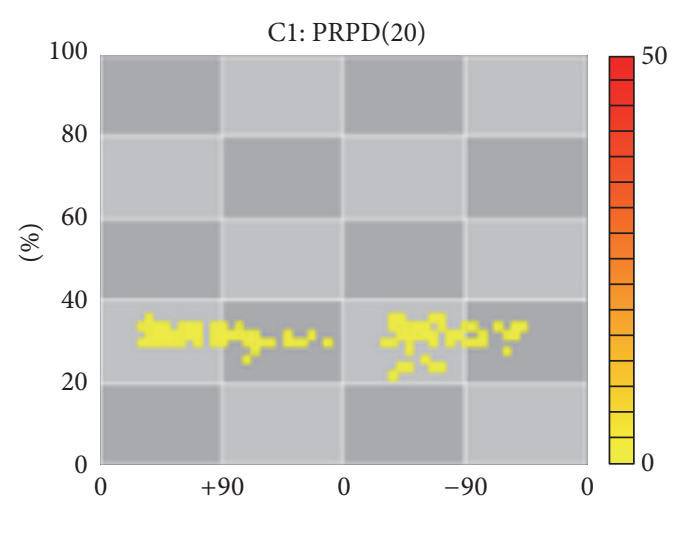

(b)

FIGURE 5: High-frequency testing results: (a) a pulse waveform; (b) PRPD spectra.

TABLE 7: The conversion of probability on sample characteristics among different classifiers.

\begin{tabular}{lccc}
\hline Defect model & C1 & C2 & C3 \\
\hline (A) Spike & 0.2936 & 0.6923 & 0.4215 \\
(B) Particulate & 0.1375 & 0.2785 & 0.4912 \\
(C) Suspended & 0.4533 & 0.3242 & 0.1884 \\
(D) Air & 0.7990 & 0.5780 & 0.7723 \\
\hline
\end{tabular}

value of $\eta_{i}$ the stronger the deterministic of classification. PD fault data of four states including spikes on the surface of high-voltage conductor, free metal particulates, suspended electrode, and a solid insulating gap take 1000 samples from each state, within which training samples change depending on the conditions, 500 test samples, and construct classifiers of three categories:

(1) Category 1: for one thousand training samples, C1 classifier is constructed in accordance with the proposed HFC PD data's diagnostic method based on information fusion and semisupervised learning.

(2) Category 2: for one thousand training samples, C2 classifier is constructed in accordance with the proposed ultrasonic signals diagnostic method based on adaptive mutation parameters of particle entropy.

(3) Category 3: for one thousand training samples, C3 classifier is constructed in accordance with the proposed UHF signal diagnostic method based on IIAART2A NN.

Table 6 shows the accuracy rates obtained when identifying four types of samples.
Fuzzy density is defined based on the accuracy of the method, and then the fuzzy density matrix is obtained as follows:

$$
\mathrm{PD}=\left[\begin{array}{lll}
0.2217 & 0.2851 & 0.3412 \\
0.2972 & 0.3361 & 0.3095 \\
0.2669 & 0.2847 & 0.3155 \\
0.3322 & 0.3182 & 0.2451
\end{array}\right]
$$

We choose a test sample as an example so the sample is identified by the $\mathrm{C} 1, \mathrm{C} 2$, and C3 classifiers and then we calculate the probability of its belonging to A, B, C, and D categories; the results are shown in Tables 7, 8, and 9 and then directly classified in Table 7; it is divided into class $\mathrm{D}$ by $\mathrm{Cl}$ and $\mathrm{C} 3$ and into A category by $\mathrm{C} 2$.

Table 8 shows the results of fuzzy measure and fuzzy integral, within which $h(x)$ is measurable function; the results of the descending corresponding item are in Table $8 ; g(\cdot)$ is the fuzzy measurement calculated according to the rank of fuzzy density and measurable function $h(x)$ and $S_{v}$ express the Sugeno fuzzy integral value of $h(x)$ and $g(\cdot)$, wherein the fuzzy density is calculated from the classification accuracy. Make judgments based on the value of $S_{v}$, and then the test sample belongs to class D. As can be seen, the fuzzy integral gets the "nonlinear" mean-value of $h(x)$. Since fuzzy measurements are from the classification accuracy of different categories, taking them as references when calculating the mean-value of multiclassification can reduce the uncertainty of multiclassifier [13].

Take PD as blurred density, to make fuzzy integral decision for various types of PD samples; recognition results are shown in Table 9; compared with Tables $4-8$, it can be 
TABLE 8: Results of fuzzy measure and fuzzy integral.

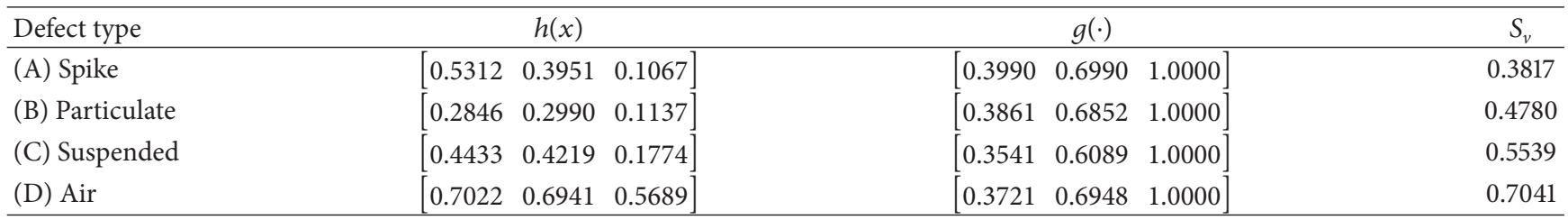
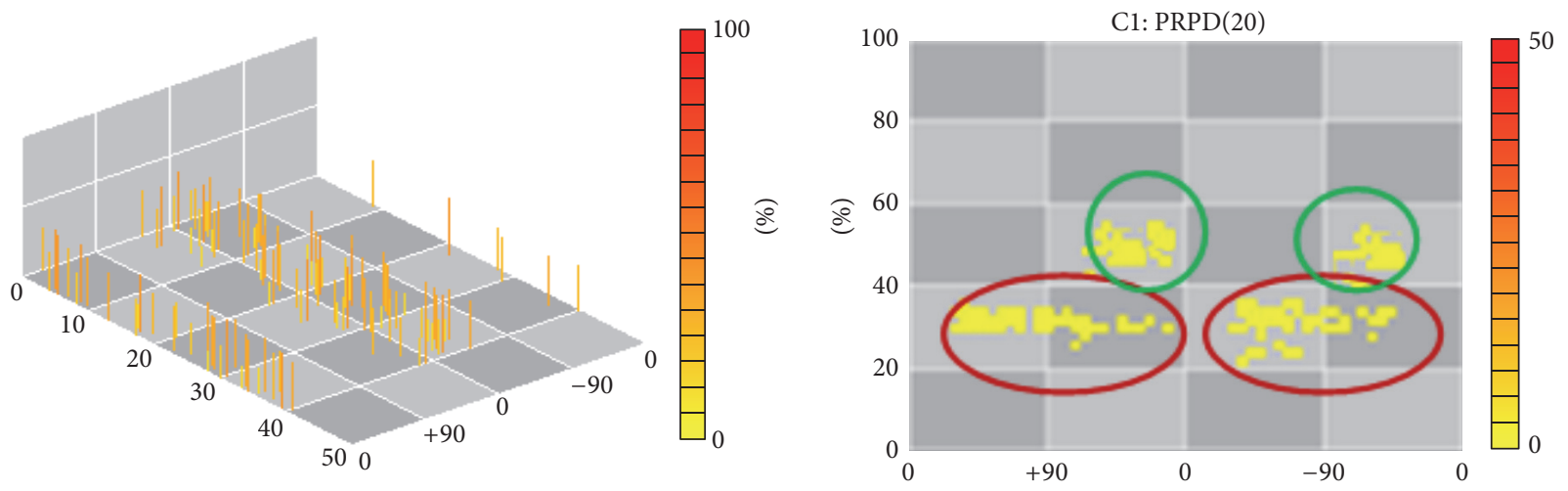

FIGURE 6: UHF testing results.

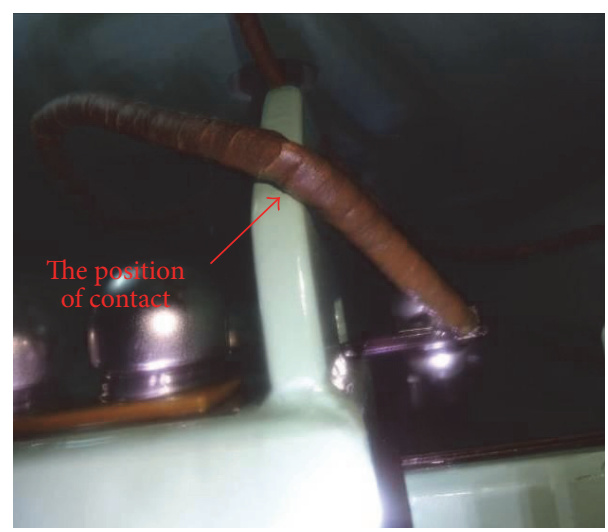

FIGURE 7: Iron core's lead insulation of column A in contact with the upper clamps.

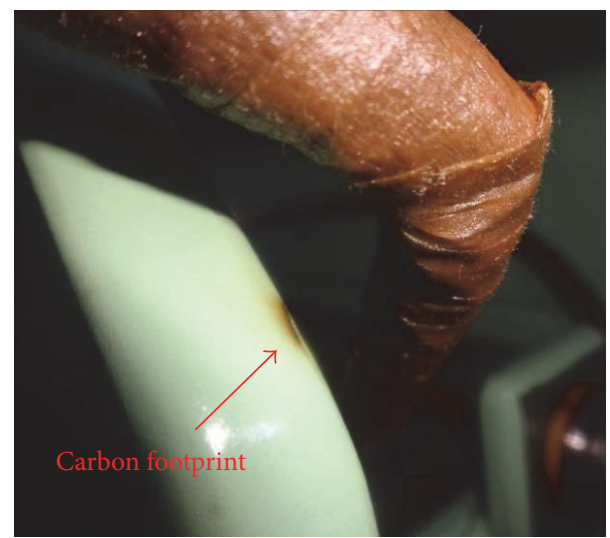

FIGURE 8: Separate the iron core's lead insulation of column A and the upper clip.
TABLE 9: Classification results of fuzzy integral fusion.

\begin{tabular}{lccc}
\hline Defect model & Test samples & Training samples & Accuracy \\
\hline (A) Spike & 1000 & 500 & $96.66 \%$ \\
(B) Particulate & 1000 & 500 & $94.37 \%$ \\
(C) Suspended & 1000 & 500 & $90.45 \%$ \\
(D) Air & 1000 & 500 & $94.50 \%$ \\
\hline
\end{tabular}

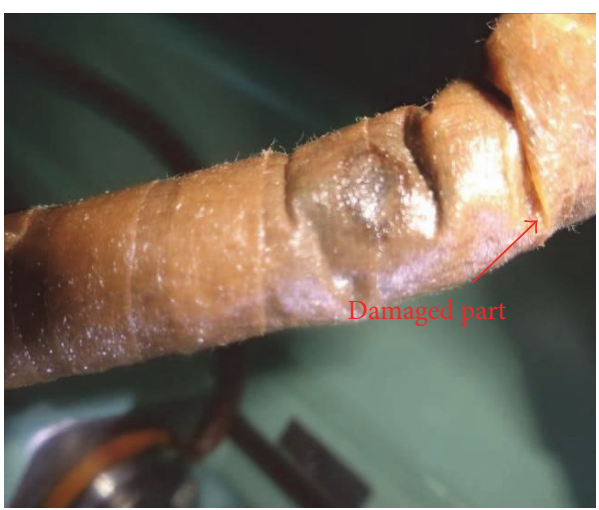

FIgURE 9: Damaged part of iron core's lead insulation of column A.

seen that the recognition accuracy of error-prone categories' sample (such as C, D) has significantly improved with fuzzy integral fusion decision. Because differences among original characteristic vectors of error-prone subsample are small, the randomness and uncertainty of recognition process make it difficult to correctly classify. Multiple classifier fusion analysis based on fuzzy integral can avoid this kind of "uncertainty" and improve the recognition accuracy to some extent. 

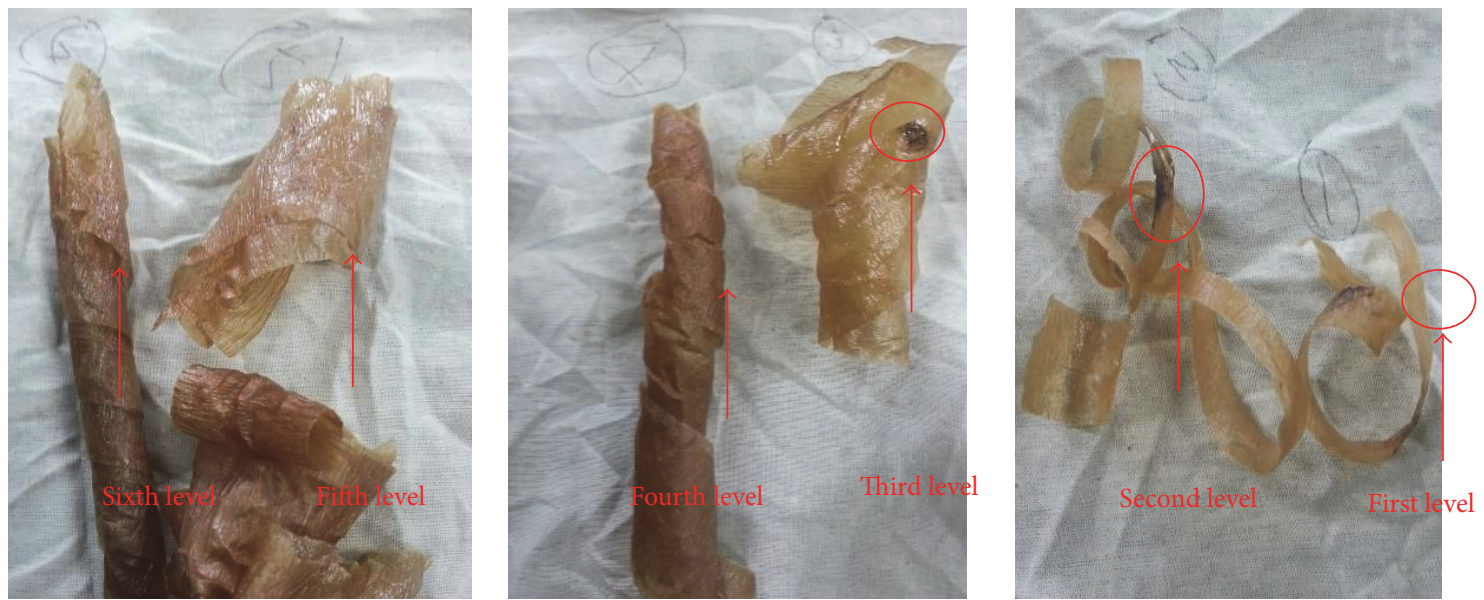

FIGURE 10: After poking iron core's lead insulation of column A.

\section{Field Application of Combined Diagnosis of PD Based on the Multidimensional Characteristic Parameters}

Detect PD on a $1000 \mathrm{kV}$ high-voltage reactor, field test with the integrated application of UHF, and ultrasonic and highfrequency method, and then implement the multiple classifier fusion analysis based on Sugeno fuzzy integral. Test results show that there are two distinct PD phenomena in the high resistance; positioning discharge source 1 is located in the upper clip part of reactor column X (the body of 1 ) and discharge source 2 is located at the bottom of the reactor winding. The accuracy of the above PD diagnosis and localization is verified by the disintegrated inspection [14].

(1) With the detecting method of high-frequency PD pulse current, it is detected that, at the clip of body 1 of the high resistance iron core clamps grounding line, the amplitude of high-frequency PD signal is the highest, reaching $500 \mathrm{mV}$; at the iron core of body 1 , the amplitude reaches $300 \mathrm{mV}$; at the clip and the iron core of body 2, the amplitude reaches $250 \mathrm{mV}$. PD phase spectra shows the characteristic of symmetric distribution in first and third quadrants, the high-frequency PD pulse current testing results are shown in Figure 5.

(2) Detecting with the UHF method, the PD detecting results are shown in Figure 6. In PRPD spectra, it is obvious that there are two discharge sources, which are shown in the red circle in the first and third quadrants and green circle in the twice and fourth quadrants, respectively.

(3) Locate the source of discharge. The discharging source is located at a position $136 \mathrm{~cm}$ away from the east side of the reactor's wall of box, $200 \mathrm{~cm}$ away from the north side of the box's wall, and at a height of $391 \mathrm{~cm}$. The discharge source is located at a middle and high position of the box, close to the east and west box's wall, and the variation trend keeps consistent with UHF detection with normalized amplitude.

(4) Significant discharge signals are detected on the reactor by the ultrasonic sensor.
(5) Two discharging points have been found in disintegrated inspection of the high resistance, and the results are consistent with the PD detection, as follows.

Discharging Point 1. Iron core's lead insulation of high resistance column A of A phase collides with the upper clamps (Figure 7); carbon footprint is found at the side of the clip (Figure 8); there are totally six corresponding lead insulation crepe papers, wherein the outer three layers of crepe paper have disruptive discharge phenomena (Figures 9 and 10).

Traces of discharge at the position of iron core's lead insulation colliding with the upper clamps may be the oil gap's PD under a high electric field (the upper end of column A is in the operation of $500 \mathrm{kV}$ voltage level, which will form a strong electric field on the outer surface of the iron core's lead insulation; and by testing the standby phase, it can be calculated that potential difference of the iron core's lead and clamp's lead of column A is significantly greater than column $\mathrm{X}$ under the rated working voltage).

Discharging Point 2. The shield cap of fixing bolt is not installed in place and collides with the fixing bolts at the bottom of the column X clamps. There exists carbon footprint (Figure 11). Because the shield cap is not installed in place, leading to being too close to clip bolts, and contact discharge happened under running vibration. The discharge here and the ultrasonic testing result are in agreement and also in agreement with the high-frequency detection result of clip and iron core ground loop anastomosis.

\section{Conclusion}

This article proposes nine PD basic characteristic parameters to reflect insulation of device and 8 phase characteristic parameters to reflect the type of discharge. The collected signals are processed with the method of high frequency, UHF, and ultrasonic. With the multiclassifier fusion method based on Sugeno fuzzy integral, integrate all kinds of defects 


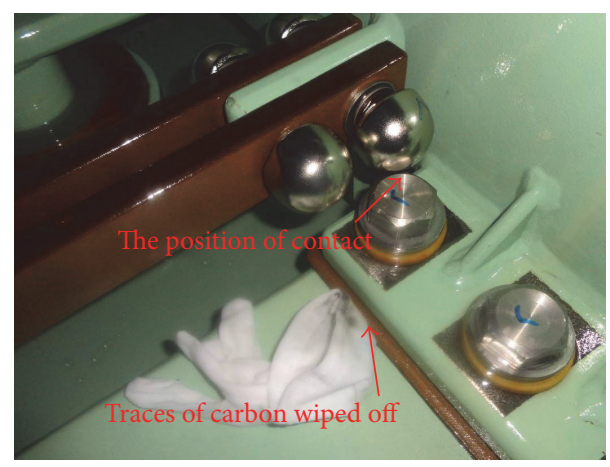

FIGURE 11: The lower lead supporting shield fixing bolts cap of high resistant's column X.

fault probability matrix, namely, the diagnosis results of high-frequency current PD data's diagnostic method based on information fusion and semisupervised learning ultrasonic signals diagnostic method based on adaptive mutation parameters of particle entropy and UHF signal diagnostic method based on IIA-ART2A NN. The results show that this method is significantly better than single detection method when diagnosing defects of spike, particulate, suspended, and air.

\section{Competing Interests}

The author declares that they have no competing interests.

\section{References}

[1] D.-S. Kim, C.-M. Hwang, Y.-N. Kim et al., "Development of an intelligent spacer built into the internal-type UHF partial discharge sensor," in Proceedings of the IEEE International Symposium on Electrical Insulation (ISEI '08), pp. 396-399, IEEE, Vancouver, Canada, June 2008.

[2] S. Kaneko, S. Okabe, M. Yoshimura, H. Muto, C. Nishida, and M. Kamei, "Detecting characteristics of various type antennas on partial discharge electromagnetic wave radiating through insulating spacer in gas insulated switchgear," IEEE Transactions on Dielectrics and Electrical Insulation, vol. 16, no. 5, pp. 14621472, 2009.

[3] R. Schwarz, M. Muhr, and S. Pack, "Partial discharge detection in oil with optical methods," in Proceedings of the IEEE International Conference on Dielectric Liquids (ICDL '05), pp. 245-248, Coimbra, Portugal, July 2005.

[4] A. I. Galushkin, Neural Networks Theory, Springer, Berlin, Germany, 2007.

[5] N. C. Sahoo, M. M. A. Salama, and R. Bartnikas, "Trends in partial discharge pattern classification: a survey," IEEE Transactions on Dielectrics and Electrical Insulation, vol. 12, no. 2, pp. 248264, 2005.

[6] W. Gao, D. Ding, and W. Liu, "Research on the typical partial discharge using the uhf detection method for GIS," IEEE Transactions on Power Delivery, vol. 26, no. 4, pp. 2621-2629, 2011.

[7] K. Wu, W. Shen, Y. Meng et al., "The reliability of multi-parameter insulation diagnosis," IEEE Transactions on Dielectrics and Electrical Insulation, vol. 17, no. 1, pp. 280-286, 2010.
[8] K. Wu, W. Shen, Y. Meng et al., "The reliability of multi-parameter insulation diagnosis," IEEE Transactions on Dielectrics and Electrical Insulation, vol. 17, no. 1, pp. 280-286, 2010.

[9] E. Gulski and F. H. Kreuger, "Computer-aided recognition of discharge sources," IEEE Transactions on Electrical Insulation, vol. 27, no. 1, pp. 82-92, 1992.

[10] T. K. Saha, "Review of modern diagnostic techniques for assessing insulation condition in aged transformers," IEEE Transactions on Dielectrics and Electrical Insulation, vol. 10, no. 5, pp. 903-917, 2003.

[11] A. Wang, Y. Liu, J. Deng et al., "Optical fiber sensor-based detection of partial discharges in power transformers," Optical Fibers and Applications, vol. 33, no. 5, pp. 305-311, 2001.

[12] J. A. Cosgrave, A. Vourdas, G. R. Jones, J. W. Spencer, M. M. Murphy, and A. Wilson, "Acoustic monitoring of partial discharges in gas insulated substations using optical sensors," IEE Proceedings A Science, Measurement and Technology, vol. 140, no. 5, pp. 369-374, 1993.

[13] M. D. Judd and O. Farish, "High bandwidth measurement of partial discharge current pulses," in Proceedings of the IEEE International Symposium on Electrical Insulation, pp. 436-439, Arlington, Tex, USA, June 1998.

[14] I. J. J. Kemp, "Partial discharge plant-monitoring technology: present and future developments," IEE Proceedings: Science, Measurement and Technology, vol. 142, no. 1, pp. 4-10, 1995.

[15] M. Boltze, S. M. Markalous, A. Bolliger et al., "On-line partial discharge monitoring and diagnosis at power cables," in Proceedings of the 76th Annual Proceeding of Electrical Insulation, 2009.

[16] M. J. Schervish, Theory of Statistics, Springer, Berlin, Germany, 1995. 


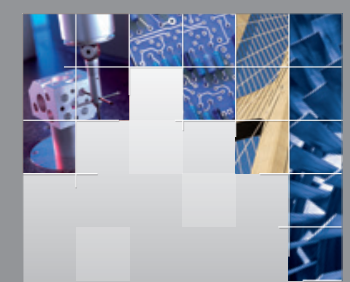

\section{Enfincering}
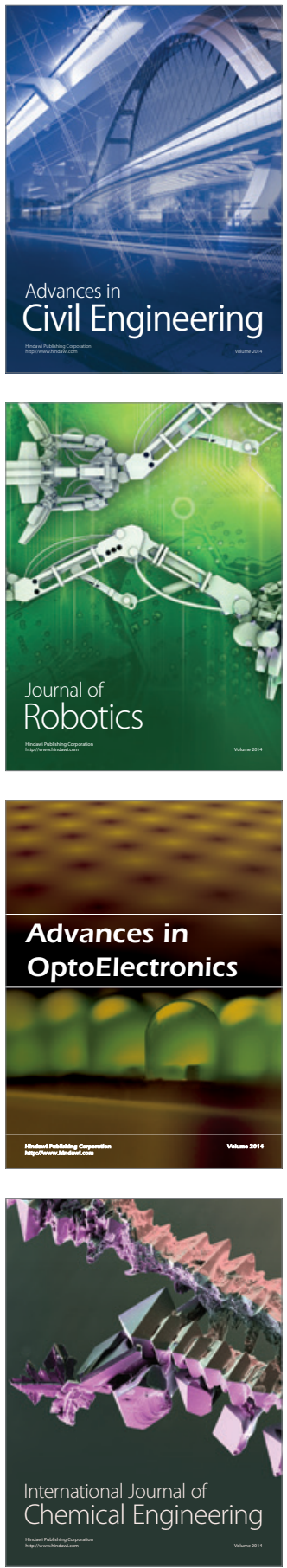

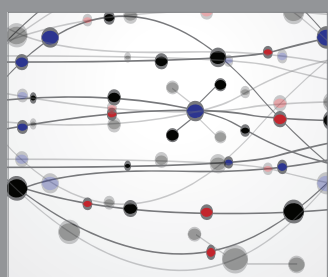

The Scientific World Journal

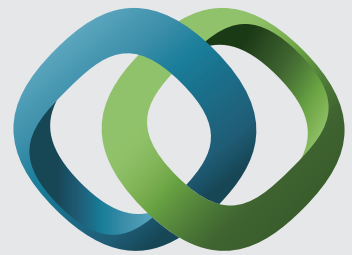

\section{Hindawi}

Submit your manuscripts at

http://www.hindawi.com
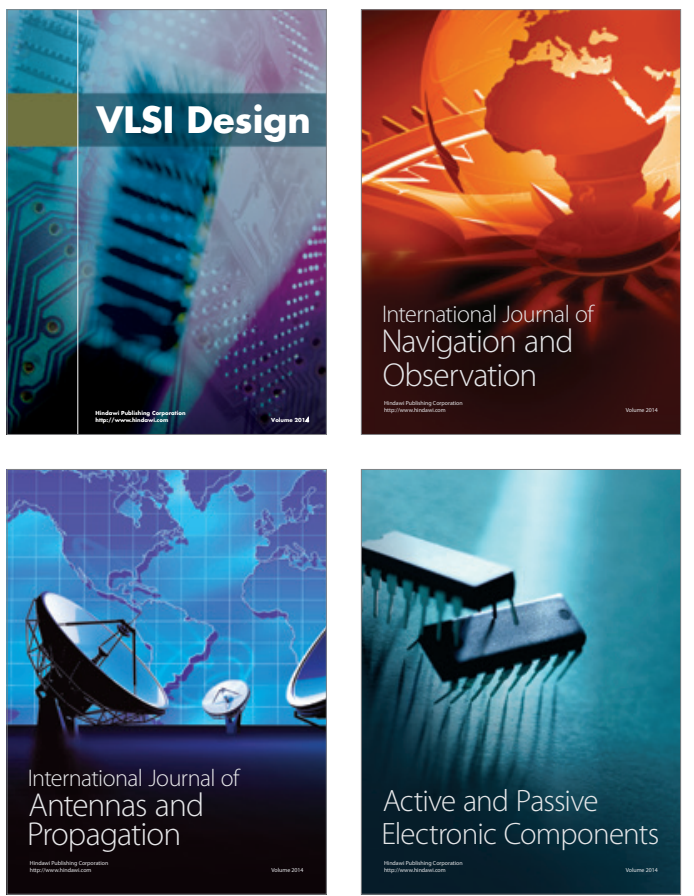
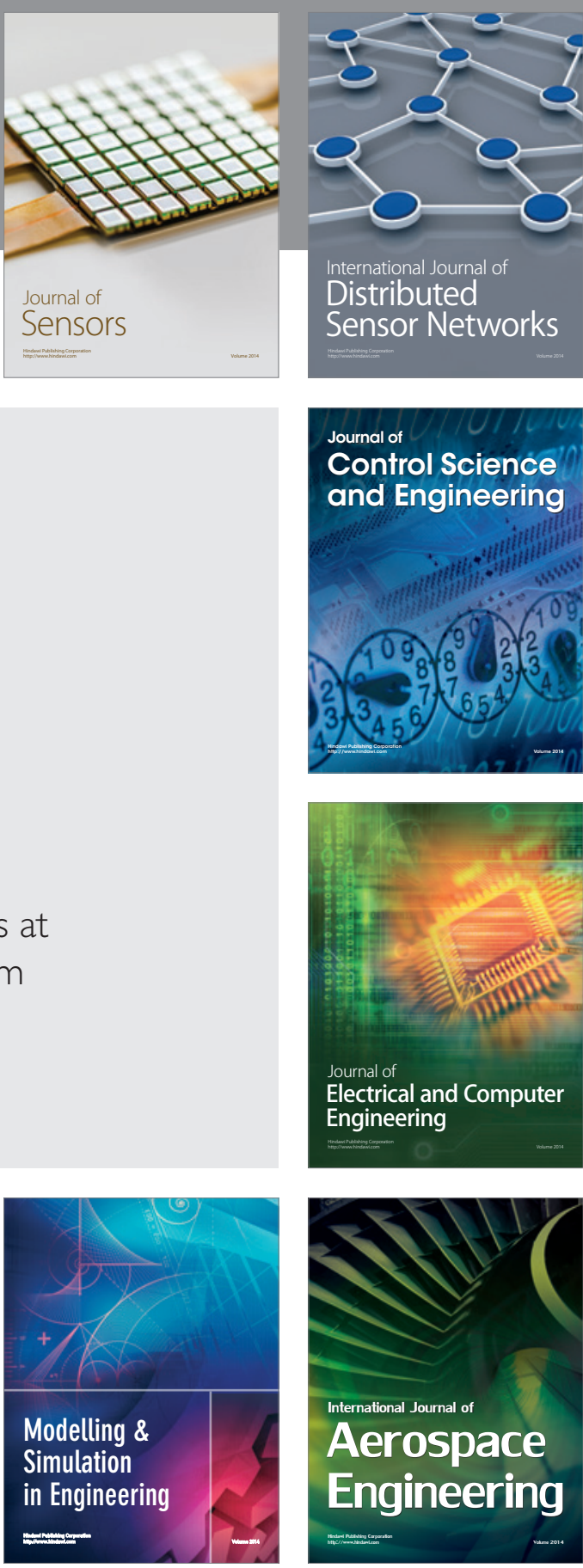

International Journal of

Distributed

Sensor Networks

Journal of

Control Science

and Engineering
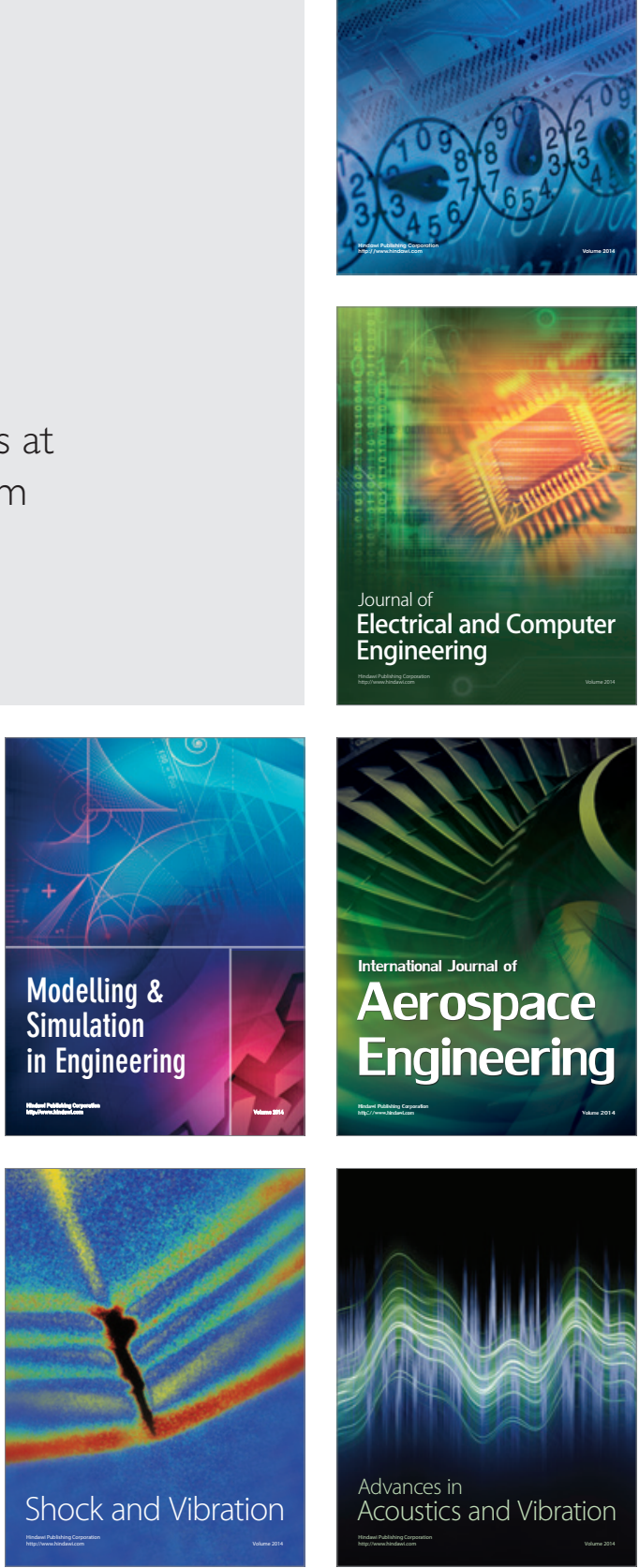